\title{
Procedure of EfFective Use of Cloudlets in WIRELESS METROPOLITAN AREA NETWORK ENVIRONMENT
}

\author{
Rashid Alakbarov and Oqtay Alakbarov \\ Institute of Information Technology, Azerbaijan National Academy of Sciences, \\ Azerbaijan
}

\begin{abstract}
The article develops a method to ensure the efficient use of cloudlet resources by the mobile users. The article provides a solution to the problem of correct use of cloudlets located on the movement route of mobile users in Wireless Metropolitan Area Networks - WMAN environment. Conditions for downloading necessary applications to the appropriate cloudlet using the possible values that determine the importance and coordinates of the cloudlets were studied. The article provides a model of the mobile user's route model in metropolitan environments and suggests a method for solving the problem.
\end{abstract}

\section{KEYWORDS}

Mobile computing clouds, cloudlet network, mobile user, route model, connection channel, metropolitan area network, virtual machine.

\section{INTRODUCTION}

Currently intensive research works are being carried out worldwide to effectively use computing and memory resources of data processing centers through cloud computing technologies. Such systems with large computing and memory resources are developed based on computer networks with high-speed connection channel. Cloud Computing technology allows to use computing and memory resources of organizations' data processing centers more effectively. Thus, Cloud Computing is an Internet service that consists of technical-software that allows using distantly located computer resources (computing and memory resources, program and data etc.) $[1,2]$.

Rapid growth of the use of mobile devices (laptops, tablets, smartphones etc.) and their connection to computing clouds via internet using their compatible telecommunication technologies (GPS, 3G/4G, Wi-Fi etc.) has prompted development of a new technology - mobile computing cloud technology.

It is known that, capabilities of any mobile device (computing and memory resources) are limited. But users use these devices to solve problems requiring great computing memory resources. For this purpose, cloud computing technologies are widely used. Thus, it is possible to eliminate the deficiency of computing and memory resources in mobile users' devices using cloud technologies [3]. Conducted researches show that, production volume of mobile equipment and number of mobile internet users is constantly increasing. The number of mobile devices to be produced in the world in 2018 will amount to 12,165 billion, while the number of mobile users will be 6,228 billion. This will increase the number of mobile cloud computing users in the near future. With the widespread use of mobile devices, there is a dramatic increase in mobile applications like surface navigation, email, web search and mobile gaming. This once again proves that mobile devices have gradually become a dominant computing platform. 
In mobile computing clouds, mobile devices connect to the computing cloud systems via Internet with the assistance of base stations and use the services they need. Currently, users are widely using three types of cloud services (IaaS, PaaS and SaaS).

The article considers the problems of balanced placement of mobile users' queries (problems or applications) taking into consideration the location and technical capabilities of cloudlets located near base stations of Wireless Metropolitan Area Networks - WMAN. Second section reviews the research on the development of network infrastructure based on cloudlets and the efficient utilization of their resources. In the third section, the issue of creating a hierarchical architecture based on cloudlets was considered. The third section reviews the issue of creating a hierarchical architecture based on cloudlets. The fourth section describes elimination of problems by placing the software used by mobile users in motion in wireless metropolitan area network environment in nearby cloudlets or those located on their movement route. In Section Five, a more efficient use of the cloudlet network resources has been proposed, which provides for the minimization of communication channels and increased reliability. Computational experiments based on the method proposed were conducted in Section Six.

\section{RELATED WORK}

Let's review the research studies on the selection of cloudlets that meet the set of requirements for the solution of the problem. Optimizing the placement of problems of the multiple mobile users that require different volume resources are among topical problems. Majority of researches in Mobile Cloud Computing technologies are dedicated to effectively organizing the processing user requests on cloud remote servers $[4,5]$. By solving user problems in nearby cloudlets, you can eliminate delays and ensure the reliable operation of the network by reducing the number of connection channels between users and cloudlets. It is also possible to reduce energy consumption, delays and disconnections by relevantly storing the applications (interface) and main parts used by the users on mobile devices and cloud servers [6]. Some researchers note that cloud servers are usually physically far from the users, which causes long-term delays in data exchange among the user and the remote cloud [7]. Close location of cloud servers to the users significantly reduces delays in data exchange [8]. Some researchers have suggested that the few number of communication channels between the user and the cloudlet perform the software in the cloudlets with high quality, minimal delay and disconnections [9].

In [10], article proposes to use cloudlets mobile application of which are located in close proximity to mobile devices in order to eliminate access restrictions to remote clouds. In [11] the article examined the mobile user's cloudlet access capabilities, the duration of communication between the user and the cloudlet, and the duration of resolution of problems.

In [12], the article reviews the solution of selection issue of virtual machines that can provide a faster resolution of the issue, based on the user's requirements, using the technical capabilities of the cloudlets and the virtual machines created in the cloudlets. In [13], the article reviews optimal balanced distribution of mobile network of mobile applications among cloud servers. Significant impact of conductivity of inter-cloudlet connection channel on rapid implementation of mobile applications was reviewed [14]. Some research works [15, 16] are dedicated to development problems of a cloudlet network infrastructure located in proximity of metropolitan wireless area network access points. It is best to create cloudlets in proximity of all Access Points of the noted network. It is not economically efficient and makes the value of created network more expensive. On the hand, it is possible that cloudlets located in proximity to certain access points are rarely used or unused. Thus, it is proposed to place more cloudlets in proximity of locations with a higher number of mobile users (malls, libraries, schools, universities, stadiums, stations, airports etc.). 
Some research works [17] analyze conditions that necessitate creation of cloudlets on mobile computing clouds and forecasting problems of placement of cloudlets near certain base stations. In [18], article reviews the issue of reducing energy consumption by optimizing the distribution of user-solved issue between the cloudlet and the remote cloud server. Some researchers have looked into the problem of quickly resolving the issue by distributing applications among several cloudlets [19]. Some authors have looked into migration of services in mobile cloud services (placing services closer to users in cloudlets) and have demonstrated some advantages of this method. A simple algorithm can be created by taking into account the frequency of inquiries to implement this process. When the need for higher-priority software is needed, a relatively less commonly used software tool can be erased from the cloud's memory resources [20]. In [21] article examined the issue of creating initial computing resources (virtual machines) using a hierarchical analysis method to effective use of computing cloud environments

\section{Creating a Hierarchically - Structured NetWork INFRASTRUCTURE AMONG CLOUDLETS}

Although the centralized clouds currently used the by users have high computing and memory resources, the processed data cannot be delivered to users at a high speed. Rapid increase in the number of mobile users in the computing cloud results in overloading of the network, causing great delays in delivering the information to the user. To eliminate the noted shortage, it is necessary to place the computing cloud resources close to the user.

Conducted research has shown that mobile Cloud Computing systems with centralized architecture are not capable of providing quality services to many users. This means that occurrence of situations such as network download, late delivery of results to the users, expensive service, delays, traffic download, etc. in such mobile computing systems, does not allow for quality service. Hierarchical (2-level) mobile Cloud Computing systems (Figure 1) are used to eliminate noted problem [22-23].

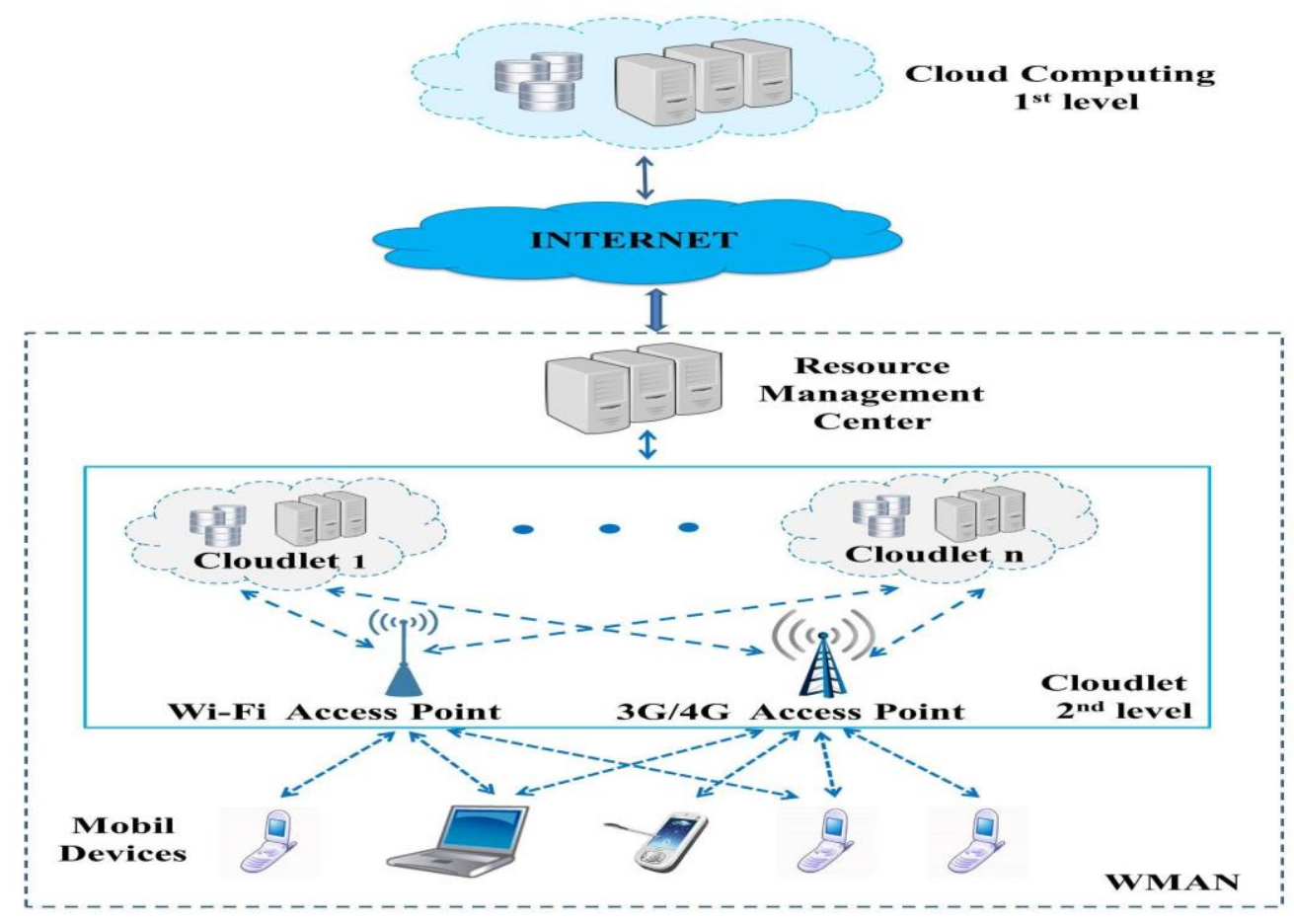

Figure 1. Architecture of hierarchically structured mobile computing clouds 
As seen in the diagram, a mobile internet user download and use the required application to the closest cloudlet system servers, this in its turn, frees the Internet network from overloading. This architecture, although partially, improves the indicators of certain parameters. Servers of Cloud Computing system are located on the 1st level; cloudlets are located near base stations in the 2nd level.

Determining which base stations are located near the base stations and what their characteristics are among topical problems. Thus, mobile servers (cloudlet) are created next to base stations of mobile operators for the wider use of mobile equipment cloud technologies. Cloudlet (small computing cloud) is a device (server) located near the users and ensures faster provision of the users with necessary information. The cloud services that users need are implemented through cloudlets, which improve the quality of services [24, 25].

Advantages of cloudlet use includes possibility of high-speed access to the service, mobility support, and reducing roaming fees. As user software applications are located in nearby cloudlets, users can instantly access information by eliminating connection delays that occur in the network. Also, as in computing clouds, technical capabilities of the cloudlets are higher than those of the mobile devices, which allows executing software applications that cannot be processed on mobile devices. Another cloudlet is selected to solve the problem when the resources of the cloudlet near the mobile user do not allow the user to solve the problem.

One of the major problems that arise when using cloudlets is the related to the connection between mobile devices and cloudlets, due to mobility of the mobile device. Sometimes a mobile user enters the area where there is no cloudlet in the coverage area of his mobile device, and in other cases there is an excessive number of cloudlets in the surroundings. In real life, mobile users visit many locations to perform office work, shop in malls and perform social activities. Most likely, there are numerous computers that can be used to create cloudlets in the noted areas. The article proposes methods and examines the conditions for downloading the necessary software applications to certain cloudlets by using the possible evaluation to determine the importance of cloudlets in order to efficiently utilize cloudlet resources that have different technical capabilities in mobile computing clouds [26].

One way to effectively utilize cloudlet resources is to preliminarily download software applications that are frequently used by mobile users (games, translation software, etc.) to the cloudlet and use them, and store those software applications there for the required period. It should be noted that placing the applications on mobile devices on clouds that are closer to them and that will be connected to them for a long period of time will allow the implementation issue to be faster and more efficient. Proximity is determined by the number of inter-cloudlet connection channels. On the other hand, applications that have high usage frequencies can be stored in cloudlets for a certain amount of time. Thus, there is no need to invoke any type of application from the remote (level 1) server. The quality of service cloudlets near base stations depends on the technical capabilities of the computer hardware used (operating speed of processors, the number of cores and virtual machines, memory, bandwidth, etc.) used in its creation. For this reason, technical capabilities of cloudlets should allow the users to download and execute software applications.

It is known that various architectures have been proposed for the efficient use of the clouds. A hierarchically structured architecture created using the Resource Manager Center has been proposed to effectively utilize cloudlet resources [27]. There is information about the location and technical capabilities of cloudlets in the RM center of the hierarchically structured network. Here, when the mobile users enter the cloud software, they first connect to the proposed RM, which then connects the user to the relevant cloud resource in the shortest period of time. MCC 
architecture is shown in Figure 1. Here, mobile devices are connected to mobile networks through the base stations (e.g., base transmission station - 3G / 4G, access point - Wi-Fi, etc.) that build and manage functional interfaces and connections (air communication) between network and mobile devices. Mobile users' inquiries and information (e.g., ID and location) are transmitted to the Resource Management Center (central processor connected to the server), which provides mobile network services. Here, mobile network operators can provide mobile users with services such as AAA (authentication, authorization and accounting) based on HA-home agent and subscriber information stored in data bases. Subsequently, the requests of the subscribers are sent to the server that communicates with the Internet from the Resource Manager of the proposed model, where it communicates with the relevant cloud over the internet. There are information about the computer hardware (server, desktop, notebook, etc.) used to create cloudlets in the management center. At the same time, the management center collects information on the technical capabilities of the cloudlets (operating speed of processors, the number of cores of the processor, number and technical characteristics, memory, etc.) and the proximity of users to which cloudlets on the mobile network. Therefore placement of invoked (selected) application from mainly cloud servers based on incoming inquiry on which cloudlet and which virtual machine that meets user requirements are among topical problems. The cloudlet Network Management Center will provide information about the cloudlet location coordinates along the movement route of the mobile user. The article is devoted to the effective placement of selected applications based on user inquiries in cloudlets located along the route.

\section{Statement Of The Problem}

No problems arise when users use the cloudlet resources in a stationary state (not in motion). For instance, the software needed by the user is downloaded and used at the nearby cloudlet. However, a problem arises in using the cloudlet resources when the user is in motion (pedestrian or machine). Cloudlets are placed near the base stations of wireless city-type mobile networks. The mobile user first downloads the software application to the nearby cloudlet.

Whenever a mobile user moves, the application used by the user is on the initially downloaded cloud. When the user reaches the necessary point moving along the route, he is connected to the initial cloudlet that stores the application using base stations located along the route and solves his or her problems. In this case, as the distance increases, the mobile user connects to the cloudlet he uses through multiple communication channels. This increases the distance between the user and the cloudlet and the number of connection channels used for connection. Thus, the mobile user in motion cannot effectively use the resources of cloudlets. The increased distance between the cloudlet and the user, and the large number of contact channels, will affect the rapid processing of the problem, increase disconnections, reduce reliability, and weaken the network bandwidth. Therefore, the article recommends a method for eliminating the noted deficiencies by placing the applications used by users in the cloudlets that are on route and in long-term connection with the users. When the user is in motion, he / she starts to move away from the base station he / she is currently using and after a certain period of time the user starts to disconnect from the base station. To ensure uninterrupted connection, the user is connected to the next nearest base station. On the other hand, the user is close to several base stations (depending on the signal area). User may only be connected with one of the base stations near him. At this connection, the closest base station is selected. The may be in short-term contact with the nearest base station, due to his / her movement route. In this case, the user connects with multiple cloudlets before reaching the destination point, based on base station proximity principle. Thus, the application is used in the process of getting from point A to Point B by loading multiple cloudlets. This leads to uploads on the transmission channels in the cloudlet network. The goal is to efficiently manage the needed application by using fewer cloudlets while the user is in motion. The route used by the user to get 
from Point A to Point B is determined by the navigational programs. Cloudlets on this route are defined. Clouds in proximity to the direction of movement are selected. The cloudlet that will remain the longest on the user's route and will resolve the issue is determined among the selected cloudlets and user's application is uploaded to selected cloudlet. Thus the user will use fewer cloudlets while moving along the route. Proposed method will help to utilize the cloud network resources more efficiently, load the connection channels less and increase the reliability.

\section{Solution Of The Problem}

Resolution of the mobile user problem within provided period depends on technical capabilities of the cloudlet. Let's assume that, cloudlet network consists of n number of cloudlets. Network resource management center has information about technical capabilities of the cloudlets (processor operation speed, number of cores, number of virtual machines, memory etc.). Computing issue of the mobile user consists of $d_{i}$ number of instructions. Let's mark computing productivity of virtual machines used in cloudlets as $S_{\tilde{i}}$. Computing period of the noted issues in selected cloudlet $C_{i}, \forall_{i}=1,2, \ldots$ will be $C_{i}(t)=\frac{d_{i}}{s_{i}}$. Let's mark the transmission speed of the channel between the mobile user and the cloud as $B_{i}$. When mobile user has to send Dout bite volume of information to the cloudlet and the cloudlet has to send Din bite volume of information back to the user, relevantly Dout/的 and $\operatorname{Din} / B_{i}$ amount of time is needed to transmit and receive information.

Let's mark the completion time of the issue as $T_{i}=\frac{C_{i}(t)}{s_{i}}+\frac{\text { Din+Dout }}{B_{i}}$, which is the sum of processing, transmitting and receiving periods of information in $C_{i}$ cloudlet [11]. At this time, we need to direct the mobile user's issue to such a cloudlet that will solve his/her issue while the mobile user is connected to the cloudlet. If the mobile user is in motion, then his/her connection period with the initial cloudlet may be shorter that resolution period of the issue. In this case, in order to continue to solving the problem, connection of the user to next cloudlet along the route of the user must be provided and process must be continued along the route until the end. The article focuses on resolution of the noted problem.

Solution of the issue can provide solution of set forward problems by being connection to the fewest number of cloudlets until reaching the final point of the route.

Structural diagram of the wireless metropolitan area networks created based on cloudlet is demonstrated in figure 2. Let's assume that, mobile user passes by multiple base stations and cloudlets of the wireless metropolitan area network as shown on Figure 2.

Coordinates of the cloudlets located in the wireless metropolitan area can be written as following:

$C_{1}\left(X_{c_{1}}, Y_{c_{2}}\right)-$ coordinates of the first cloudlet;

$C_{2}\left(X_{\mathrm{C}_{2}}, Y_{\mathrm{c}_{2}}\right)$ - coordinates of the second cloudlet;

$\dddot{C_{n}}\left(X_{c_{n}}, Y_{c_{n}}\right)$ - coordinates of the Nth cloudlet.

Mobile user can write the coordinates of the points along the route as following:

$M\left(X_{m 1}, Y_{m 1}\right)$ - Coordinates of the first point of the route;

$M\left(X_{m 2}, Y_{m 2}\right)$ - Coordinates of the second point of the route;

$\cdots$

$M\left(X_{m K}, Y_{m K}\right)$ - Coordinates of the Kth point of the route; 


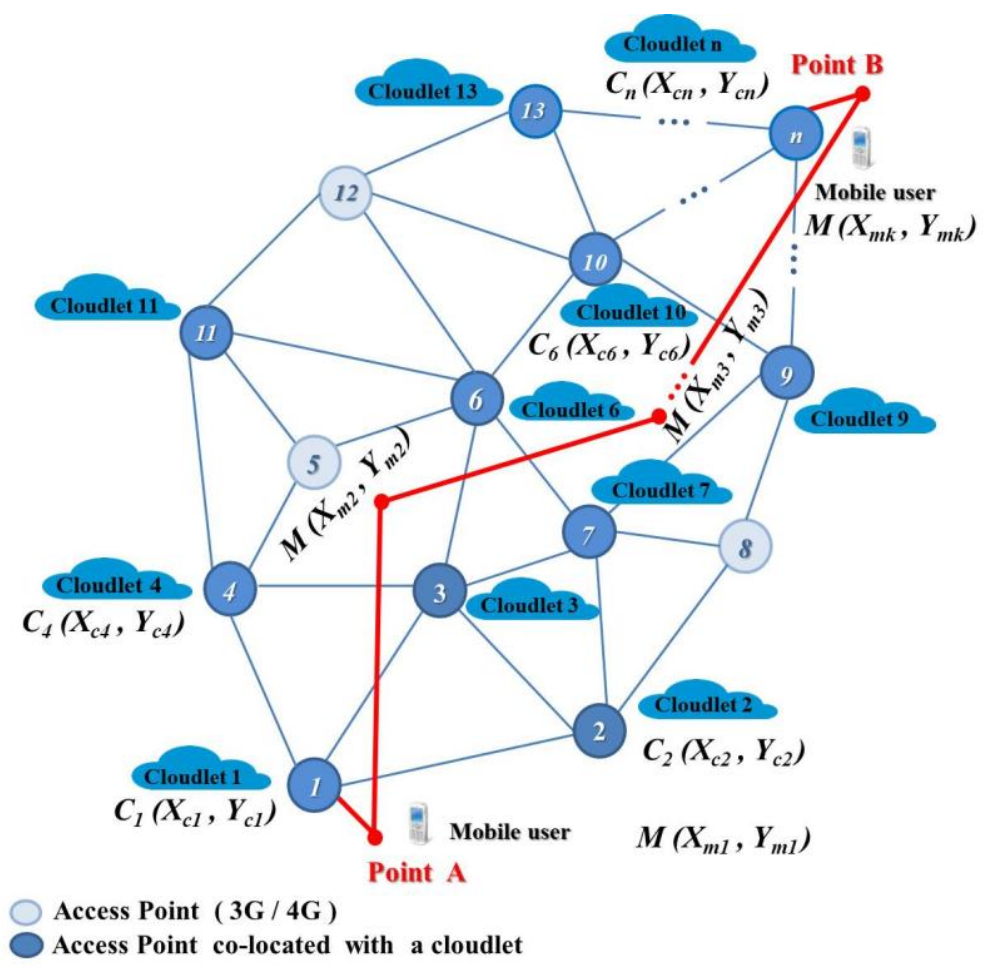

Figure 2. Route of the mobile user in wireless metropolitan area network

It is known that, if the mobile user remains at any point of the route, as well as the first point of the route for a lengthy period of time, he can solve his problems without creating a new connection with another cloudlet, while being connected to one (nearest) cloudlet. If the mobile user will remain at the kth point of the route for a long period of time, then he can form a connection with a cloudlet located at the shortest distances from the cloudlet of this point. If mobile user's connection to the connected cloudlet is interrupted during movement, he can connect to the near cloudlet that meets certain requirements. Let's mark the locations (coordinates) of the mobile user $(M)$ and cloudlet $\left(C_{i}\right)$ with $M\left(X_{m j_{1}}, Y_{m j}\right)$ and $C\left(X_{C n}, Y_{C n}\right)$ respectively. Let's mark the transmitting range of the mobile device with $r$. Connection of mobile user to $C_{i}$ cloudlet in $t$ period is only possible when $\left\|M\left(V_{m i}, Y_{m i}\right)-C\left(X_{C n}, Y_{C n}\right)\right\| \leq r$ condition is met. Here, $\|\times\|$ is the Euclidean norm in 2 Dimension. This norm is widely used to measure the distance between points in dual dimension systems.

For example, distance between any jth point along the route and nth cloudlet is determined as following [11].

$$
d_{i n}=\left\|M\left(X_{m i}, Y_{m i}\right)-C\left(X_{C n}, Y_{C n}\right)\right\|=\sqrt{\left(X_{m i}-X_{C n}\right)^{2}+\left(Y_{m i}-Y_{C n}\right)^{2}}
$$

The shortest distance between the $\mathrm{j}$ point of the route and the cloudlets can be determined as following.

$$
d_{\min }=\min \left\{d_{j n}\right\}, \quad n \in[1, N]
$$


Such $\mathrm{r}$ (signal reception range of the mobile device) distance can be determined within the network environment shown in Figure 2, in case of $d_{j n} \leq r$ at this point mobile user can create a connection with determined cloudlet or created connection can continue, otherwise a connection cannot be formed or created connection can interrupt.

But connecting to the nearest cloudlet at every disconnection moment cannot always provide achieving set objectives (connection of the most connected cloudlet timewise). The issue can be solved as following. Portions of the route can be imagined as segments and the entire route can be imagined as a sum (connection) of straight segments. The route depicted in Figure 2 can be presented as following formula:

$$
M=U\left[M\left(X_{m(k-1)}, Y_{m(k-1)}\right), M\left(X_{m k}, Y_{m k}\right)\right]
$$

In this formula, there are K-1 number of segments and $k \in[2, K]$.

For the solution of the problem, we can assume that there are one or more cloudlets that can connect with the mobile user can at any point on the route:

$$
\forall M\left(X_{m j}, Y_{m j}\right) \in M, \exists C\left(X_{C n}, Y_{C n}\right) \Rightarrow d_{j n} \leq r
$$

Straight line formula passing through each segment can be written as following:

$$
\frac{x-X_{m(k-1)}}{X_{m k}-X_{m(k-1)}}=\frac{y-Y_{m(k-1)}}{Y_{m k}-Y_{m(k-1)}}
$$

It is known that, such formulation of the straight line formula can be written within $Y_{m k} \neq Y_{m(k-1)}$ and $X_{m k} \neq X_{m(k-1)}$ conditions. If we simplify this formula, then we will obtain the formula of a straight line that is passing through two points and general:

$$
\left(Y_{m k}-Y_{m(k-1)}\right) x-\left(X_{m k}-X_{m(k-1)}\right) y+\left(X_{m k} * Y_{m(k-1)}-Y_{m k} * X_{m(k-1)}\right)=0
$$

If $X_{m k} \neq X_{m(k-1)}$ condition is met, (4) straight line can be written as following equation with angle coefficient:

$y=\frac{\left(Y_{m(k-1)}-Y_{m k}\right)}{\left(X_{m k}-X_{m(k-1)}\right)} * x+\frac{\left(X_{m k} * Y_{m(k-1)}-Y_{m k} * X_{m(k-1)}\right)}{X_{m k}-X_{m(k-1)}}$

straight line equation within $Y_{m k}=Y_{m(k-1)}$ and $X_{m k} \neq X_{m(k-1)}$ conditions

Short distance from $y=Y_{m k}$ and $C\left(X_{C n}, Y_{C n}\right)$ cloudlet until the straight line will $\mathrm{be}^{d_{k n}}=a b s\left(Y_{m k}-Y_{C n}\right)$.

Straight line equation within $Y_{m k} \neq Y_{m(k-1)}$ and $X_{m k}=X_{m(k-1)}$ conditions

Short distance from $x=X_{m k}$ and $C\left(X_{C n}, Y_{C n}\right)$ cloudlet until the straight line will $\mathrm{be}^{d_{k n}}=a b s\left(X_{m k}-X_{C n}\right)$. 
If the velocity of the mobile is $v(t)$ at any $t$ point of its movement, after passing of $t$ amount of time from the start of the movement, this user will be at $M\left(X_{m}(t), Y_{m}(t)\right)$ point. As this point belongs to the route

$M\left(X_{m}(t), Y_{m}(t)\right) \in M$

And coordinates of the point can be determined as following.

$$
\begin{aligned}
& X_{m}(t)=X_{m 1}+\int_{0}^{t} v_{x}(t) d t \\
& Y_{m}(t)=Y_{m 1}+\int_{0}^{t} v_{y}(t) d t
\end{aligned}
$$

In formulas (5)-(6), $v_{x}(t)$ and $v_{y}(t)$ are the components of the movement velocity of the mobile user along the coordinate axes. Here $\sqrt{\left(v_{x}(t)\right)^{2}+\left(v_{y}(t)\right)^{2}}=v(t)$ equality is met.

As the metropolitan area route consists of the sum of straight-line segments, integrals in (5)-(6) formulas can be replaced with relevant sums.

Let's mark the cloudlet set that allows forming a connection with first the first of the route at the beginning of the movement, i.e. setting of cloudlets that meet

$$
\sqrt{\left(X_{m 1}-X_{C n}\right)^{2}+\left(Y_{m 1}-Y_{C n}\right)^{2}} \leq r
$$

Conditions with $\mathrm{C}(0)$, and mark the set of cloudlets that can form a connection with $M\left(X_{m}(t), Y_{m}(t)\right)$ point at any $t$ moment of the movement and meets

$$
\sqrt{\left(X_{m}(t)-X_{C n}\right)^{2}+\left(Y_{m}(t)-Y_{C n}\right)^{2}} \leq r
$$

Conditions with $\mathrm{C}(\mathrm{t})$. At the beginning of the movement, cloudlet must be selected in such manner that, connection with the cloudlet could last for a long period of time. For this purpose cloudlet or one of the cloudlets can meet

$$
\left\{\begin{array}{c}
C(0) \cap C(t-1) \neq \emptyset \\
C(0) \cap C(t)=\emptyset
\end{array}\right.
$$

Conditions must be selected. This cloudlet determines long-term connection at the beginning of the movement.

A cloudlet that provides long-term connection during the remaining second part of the route from point $M\left(X_{m}(t), Y_{m}(t)\right)$ of the route must be selected. If we move as in the starting point, let's mark the set of cloudlets that can form a connection with $M\left(X_{m}(\tau), Y_{m}(\tau)\right)$ at which the mobile can be at any $\tau>t$ moment as $C(\tau)$. And this set consists of cloudlets that meet

$$
\sqrt{\left(X_{m}(\tau)-X_{C n}\right)^{2}+\left(Y_{m}(\tau)-Y_{C n}\right)^{2}} \leq r
$$

Conditions. The cloudlet that provided long-term connection during the second part of the route must meet following conditions: 


$$
\left\{\begin{array}{c}
C(t) \cap C(\tau-1) \neq \emptyset \\
C(t) \cap C(\tau)=\emptyset
\end{array}\right.
$$

Shown operations are carried out until the end of the movement of the mobile user along the route and cloudlets that provide a long-term connection at any part of the movement are selected. Sequence of selected R number of cloudlets can be marked as

$$
C=\left\{C_{\max }(1), C_{\max }(2), \ldots C_{\max }(R)\right\} .
$$

It is known that this sequence can be determined at the beginning of the movement using the movement route information of the mobile user and set of cloudlets in metropolitan environment. Sequence can be modified if information about technical conditions of the cloudlets changes during the movement. It should be noted that the found solution allows the mobile user to use a minimum number of cloudlets for connection while moving to the final point of the route. At the same time, the given method enhances the efficiency of use of cloudlet resources by establishing connection with a few cloudlets. Thus, frequent establishment of frequent connections and disruption of connection leads to the inefficient use of cloudlet resources.

\section{EXPERIMENT}

Computing experiments on developed model was implemented using following data. Here, all measurements are provided in proportion to $r$ distance. Movement route of mobile users surrounded with cloudlets is depicted in Figure 3.

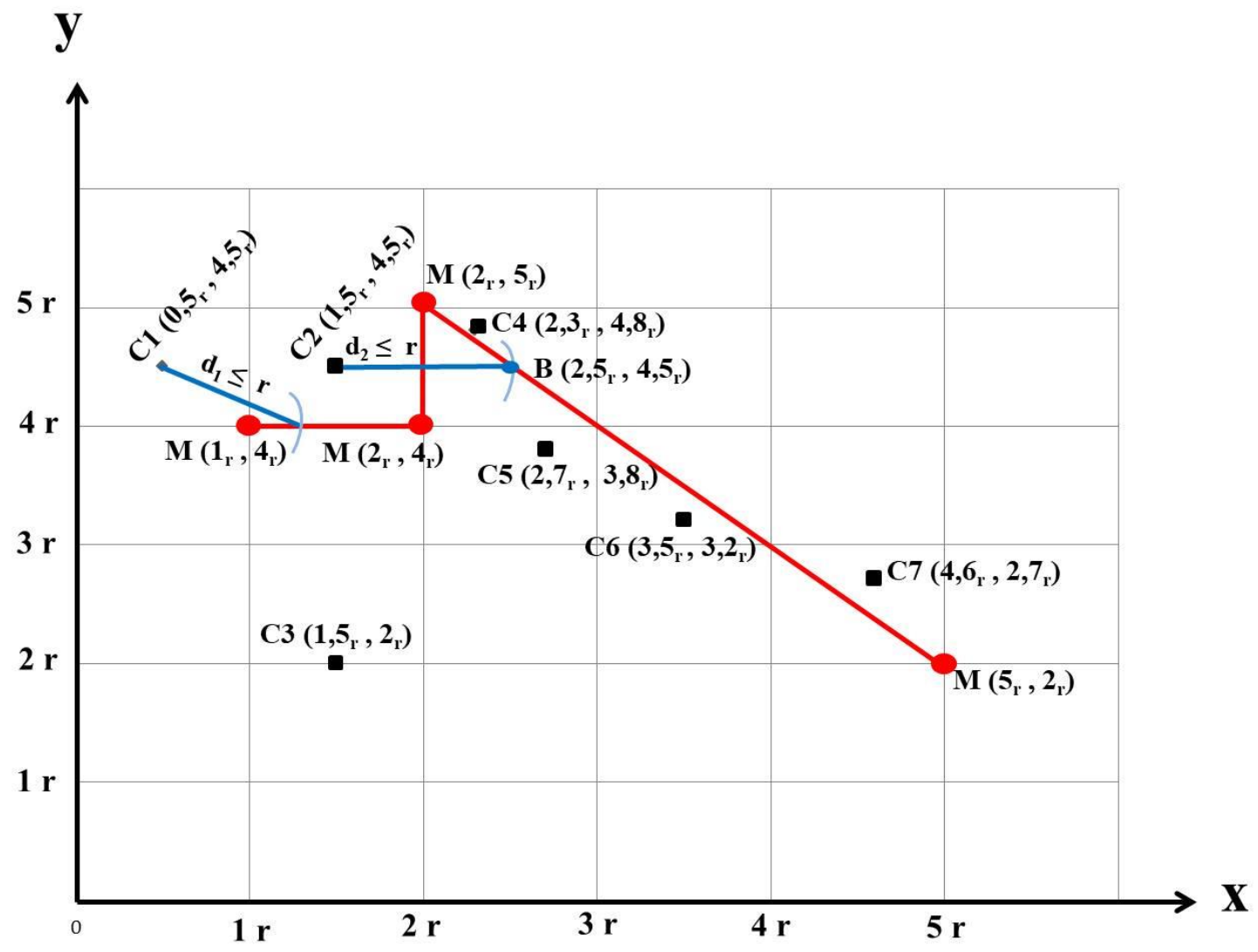

Figure 3. Movement route of the mobile user in cloudlet network 
International Journal of Computer Networks \& Communications (IJCNC) Vol.11, No.1, January 2019

Points along the movement route of the mobile user:

$M(r, 4 r), M(2 r, 4 r), M(2 r, 5 r), M(5 r, 2 r)$.

Coordinates of the cloudlets:

$C 1(0,5 r, 4,5 r), C 2(1,5 r, 4,5 r), C 3(1,5 r, 2 r), C 4(2,3 r, 4,8 r), C 5(2,7 r, 3,8 r)$, $C 6(3,5 r, 3,2 r), C 7(4,6 r, 2,7 r)$.

Let's assume that the mobile user moves at a stable speed of $v=0,2 r$. For this reason, we can present time-coordinate dependence since the beginning of the movement using following formula:

$x(t)=\left\{\begin{array}{lc}r+v t ; & 0 \leq t<5 \\ 2 r ; & 5 \leq t<10 \\ 2 r+(t-10) v * 0,71 ; & 10 \leq t<25\end{array}\right.$
$y(t)=\left\{\begin{array}{cc}4 r ; & 0 \leq t<5 \\ 4 r+(t-5) v ; & 5 \leq t<10 \\ 5 r-(t-10) v * 0,71 ; & 10 \leq t<25\end{array}\right.$

If the nearest cloudlet to the M1(r,4r) point of the route is selected at the beginning of the movement, then it is $\mathrm{C} 1(0,5 \mathrm{r} ; 4,5 \mathrm{r})$ cloudlet.

The distance between this route point and the cloudlet is

$d_{1}=\sqrt{(r-0,5 r)^{2}+(4 r-4,5 r)^{2}}=\sqrt{0,5} r<r$ and it is the shortest distance.

The second cloudlet that is close to the starting point of the movement and with which we can establish a connection is $\mathrm{C} 2(1,5 \mathrm{r} ; 4,5 \mathrm{r})$.

the distance from these cloudlets to the starting point of the movement is

$d_{2}=\sqrt{(1,5 r-r)^{2}+(4 r-4,5 r)^{2}}=\sqrt{0,5} r<r$ and meets $d_{1}=d_{2}$ condition. It must be noted that, cloudlets that belong to $\mathrm{C}(0)$ set consists of these two cloudlets:

$C(0)=\{\mathrm{C} 1(0,5 \mathrm{r} ; 4,5 \mathrm{r}) ; \mathrm{C} 2(1,5 \mathrm{r} ; 4,5 \mathrm{r})\}$.

But, if the first cloudlet is selected, connection will last until the first t period that meets the $\sqrt{\left(0,5 r-(r+0,2 r t)^{2}+(4 r-4,5 r)^{2}\right.} \geq r$

Inequality.

As both sides of the inequality are greater than 0 , we can increase both sides to the square to solve it:

$(0,5 r-(r+0,2 r t))^{2}+(4 r-4,5 r)^{2} \geq r^{2}$

If we simplify it, we obtain:

$0,25 r^{2}-0,2 r^{2} t+0,04 r^{2} t^{2}+0,25 r^{2} \geq r^{2}$ 
If we transfer all components to the left and divide both sides by $0,04 r^{2}$, we will obtain

$$
t^{2}+5 t-12,5 \geq 0
$$

If we split into multipliers:

$$
(t+2,5+\sqrt{18,75})(t+2,5-\sqrt{18,75}) \geq 0
$$

Here, we obtain the following systems of inequalities:

$$
\begin{array}{r}
\left\{\begin{array}{l}
t+2,5+\sqrt{18,75} \geq 0 \\
t+2,5-\sqrt{18,75} \geq 0
\end{array}\right. \\
\left\{\begin{array}{l}
t+2,5+\sqrt{18,75} \leq 0 \\
t+2,5-\sqrt{18,75} \leq 0
\end{array}\right.
\end{array}
$$

Solution of the first system

$$
t \geq \sqrt{18,75}-2,5
$$

Solution of the second system

$$
t \leq-\sqrt{18,75}-2,5
$$

As $t \geq 0$ in our problem, then

$t=\sqrt{18,75}-2,5=2,5(\sqrt{3}-1)$

is the solution we are interested in.

After the $t_{1}=2,5(\sqrt{3}-1)$ value of $t$, which is the solution of this in quality, connection is disrupted and new cloudlets must be found. The point which is relevant to the shown value of $t_{1}$ is located at the first part of the route (Figure 3).

The second cloudlet provides a connection until the B $(2,5 \mathrm{r}, 4,5 \mathrm{r})$ point located in the third part of the route (Figure 3). The distance until this point of the route from the starting point of the movement is greater and for this reason, this cloudlet (mobile user will be connected to this cloud for a long period of time) must be selected for connection.

Effectiveness of established method is clear from this point. Afterwards, other cloudlets that provide long-term connection can be found applying the analogical algorithm.

\section{Conclusions}

The article provides a solution for the issue of correct use of cloudlets located along the route of mobile user in motion in Wireless Metropolitan Area Networks (WMAN). For this purpose, we provided a model of the mobile users' route consisting of straight line segments located in 
cloudlet network. A method for selecting the sequence of cloudlets that can provide long-term connection while moving along this route is developed. This sequence (set of cloudlets) can be determined at the start of the movement using the information about movement route of the mobile user. Algorithm can be applied starting at any point of the route. This allows to repeatedly apply the algorithm in necessary cases. Proposed method assists to use the cloudlet network resources more efficiently, load the connection channels less and increase the reliability.

In the future, the solution to the problem of selecting virtual machines in cloudlets located along the movement routes will be reviewed for faster execution of software applications by mobile users. As mobile users of the cloudlet network are moving, the solution of the problem that provides less loading of connection channels of the network by using less cloudlet will be reviewed.

\section{REFERENCES}

[1] R.M. Alguliyev, R.K. Alekperov, "Cloud Computing: Modern State, Problems and Prospects", Telecommunications and Radio Engineering, vol.72, no.3, 2013, pp.255-266.

[2] H. T. Dinh, C. Lee, D. Niyato, P. Wang, "A survey of mobile cloud computing: Architecture, applications, and approaches," Wireless Communications and Mobile Computing, vol.13, no.18, 2013, pp.1587-1611.

[3] T. Diaby, B.B. Rad, "Cloud Computing: A review of the Concepts and Deployment Models", International Journal of Information Technology and Computer Science, vol.9, no.6, 2017, pp.50-58.

[4] R. Alakbarov, F. Pashayev, M. Hashimov, "Development of the Method of Dynamic Distribution of Users' Data in Storage Devices in Cloud Technology," Advances in Information Sciences and Service Sciences, vol.8, no.1, 2016, pp.16-21.

[5] O.P. Akomolafe, M.O. Abodunrin, "A Hybrid Cryptographic Model for Data Storage in Mobile Cloud Computing," I. J. Computer Network and Information Security, no.6, 2017, pp.53-60.

[6] Y.C. Shim, "Effects of cloudlets on interactive applications in mobile cloud computing environments," International Journal of Advanced Computer Technology, vol.4, no.1, 2015, pp. 5462 .

[7] M.Satyanarayanan, P.Bahl, R.Caceres, N.Davies, "The case forvm-based cloudlets in mobile computing," Pervasive Computing, IEEE, vol.8, no.4, 2009, pp.14-23.

[8] K. Ha, P. Pillai, W. Richter, Y. Abe, M. Satyanarayanan. "Just-in-time provisioning for cyberforaging," in Proceeding of the 11th annual international conference on Mobile systems, applications, and services. ACM, 2013, pp.153-166.

[9] Y.C. Shim, "Effects of cloudlets on interactive applications in mobile cloud computing environments," International Journal of Advanced Computer Technology, vol.4, no.1, 2015, pp.54-62.

[10] H. Qi, A. Gani, "Research on Mobile Cloud Computing: Review, Trend and Perspectives," İEEE 2nd International Conference on Digital Information and Communication Technology and its Applications, 2012, pp. 195-202.

[11] Y.Li ,W. Wang, "The Unheralded Power of Cloudlet Computing in the Vicinity of Mobile Devices," IEEE Global Communications Conference (GLOBECOM), 2013, pp. 4959-4964. 
[12] R.K. Alekberov, F.H. Pashayev, O.R. Alekperov, "Effective Use Method of Cloudlet Resources by Mobile Users," 11th IEEE International Conference on Application of Information and Communication Technologies. Moscow, 2017, pp. 401-403.

[13] M.Jia, W.Liang, Z.Xu, M.Huang, "Cloudlet load balancingin wireless metropolitanarea networks," IEEE, 10-14 April, 2016, pp. 730-738.

[14] C. Shi, V. Lakafosis, M.H. Ammar, E.W. Zegura, "Serendipity: enabling remote computing among intermittently connected mobile devices," in Proc. of the ACM MobiHoc, 2012, pp.145-154.

[15] R.G. Alakbarov, F.H. Fahrad, O.R. Alakbarov, "Forecasting Cloudlet Development on Mobile Computing Clouds,” I.J. Information Technology and Computer Science, no.11, 2017, pp.23-34.

[16] D. Sarddar, R. Bose, "A Mobile Cloud Computing Architecture with Easy Resource Sharing," International Journal of Current Engineering and Technology. vol.4, no.3, 2014, pp.1249-1254.

[17] M. Jia, J. Cao, W. Liang, "Optimal Cloudlet Placement and User to Cloudlet Allocation in Wireless Metropolitan Area Networks,” IEEE Transactions on Cloud Computing, vol.5, no.4, 2017, pp.725737.

[18] E. Gelenbe, R. Lent, M. Douratsos, "Choosing a local or remote cloud," Proceedings of 2nd International Symposium on Network Cloud Computing and Applications, 2012, pp.25-30.

[19] T. Verbelen, P. Simoens, F. D. Turck, and B. Dhoedt, "Cloudlets: Bringing the cloud to the mobile user," Proceedings of 3rd workshop on Mobile Cloud Computing and Services, ACM, 2012. pp.2936 ,

[20] F. Liu, P. Shu, H. Jin, L. Ding, J. Yu, D. Niu, B. Li, "Gearing resource-poor mobile devices with power fulclouds: architectures, challenges, and applications," IEEE Wireless Communications. vol.20, no.3, 2013, pp.14-22.

[21] A.A. Mikryukov. R.I. Hantimirov, "The task of the initial allocation of resources in cloud computing environments based on the analytic hierarchy process," Applied Informatics. no.8, 2015, pp.184-185.

[22] Z. Xu, W. Liang, W. Xu, M. Jia, S. Guo, "Efficient Algorithms for Capacitated Cloudlet," Placements. IEEE Transactions on Parallel and Distributed Systems, vol.27, no.10, 2016, pp. 28662880.

[23] P. Gupta, S. Gupta, "Mobile Cloud Computing: The Future of Cloud," International Journal of Advanced Research in Electrical, Electronics and Instrumentation Engineering. vol.1, no.3, 2012, pp.134-144.

[24] M. Goyal, S. Singh, "Mobile Cloud Computing," International Journal of Enhanced Research in Science Technology\& Engineering, vol.3, no.4, 2014, pp. 517-521.

[25] T. Diaby, B.B. Rad. "Cloud Computing: A review of the Concepts and Deployment Models," International Journal of Information Technology and Computer Science, vol. 9, no. 6, 2017, pp. 5058.

[26] G. Huerta-Canepa, D. Lee, “A virtual cloud computing provider for mobile devices,” International Journal of Advance Research, Ideas and Innovations in Technology. Vol. 3, issue3, 2017, pp. 411414.

[27] D. Sarddar, R. Bose, "A Mobile Cloud Computing Architecture with Easy Resource Sharing," International Journal of Current Engineering and Technology. vol.4, no.3, 2014, pp.1249-1254. 


\section{AUTHORS}

Dr. Rashid Alakbarov graduated from-Automation and Computer Engineering\| faculty of Azerbaijan Polytechnic University named after C.Ildirim. He received his $\mathrm{PhD}$ degree in 2006 from Supreme Attestation Commission under the President of the Republic of Azerbaijan. His primary research interests include various areas in cloud computing, data processing, computer networks, virtual computing, particularly in the area of distributed computing. He is head of department at the Institute of Information Technology as of 2002. Since 2010, he has been leading the development of "AzScienceNet" infrastructure. In 2011, he was appointed a deputy director of the institute by the decision of the Presidium of Azerbaijan National Academy of Sciences. He is the author of 85 scientific papers, including 5 inventions.

Mr. Oqtay Alakbarov is PhD student of Institute of Information Technology of Azerbaijan National Academy of Sciences. His primary research interests include various areas in cloud computing, mobile cloud computing, mobile technologies, particularly in the area of cloud technology applications. He is the author of 3 journal scientific papers and 3 proceedings.
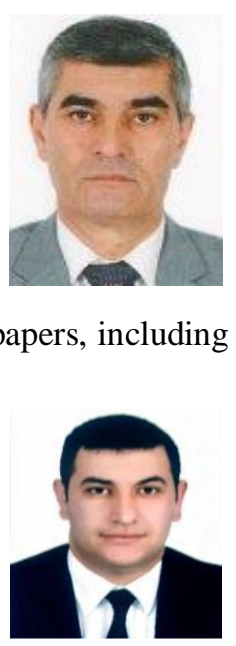